\title{
VARIATIONAL METHODS FOR PDES APPLIED TO STOCHASTIC PARTIAL DIFFERENTIAL EQUATIONS
}

\author{
GJERMUND VÅGE
}

\section{Introduction.}

During the last couple of years there has been a growing interest in stochastic partial differential equations (SPDEs). Various methods have been used to study SPDEs, see [10] and the references therein. Here we apply white noise analysis to obtain abstract existence and uniqueness theorems. More specifically we combine the ideas of Kondratiev spaces with variational methods for partial differential equations. We show that this approach applies to elliptic, parabolic, as well as hyperbolic SPDEs.

To illustrate our ideas on elliptic SPDEs, we prove in Section 4 that there exists a unique solution, $u$, satisfying

$$
\begin{gathered}
-\nabla \cdot(F \diamond \nabla u)=f \text { for } x \in D \\
\left.u\right|_{\partial D}=\left.g\right|_{\partial D},
\end{gathered}
$$

where $F, f$, and $g$ are given stochastic processes and $\diamond$ denotes the Wick product. If $F$ is the Wick exponential of smoothed white noise, $F=\exp ^{\diamond} W_{\phi_{x}}$, we obtain the pressure equation in a stochastic medium, first solved in [6]. This equation is a model for flow in an (stochastic) isotropic porous medium where $F$ is the permeability.

If $F, f$ are deterministic and $g=0$, the Wick product coincides with the ordinary product and existence of a unique variational solution of the deterministic problem (1)-(2) can be shown as follows. We apply both sides of (1) to a test function $v \in H=H_{0}^{1}(D)$ and integrate by parts to obtain the variational problem

$$
\text { find } u \in H \text { such that } b(u, v)=L(v) \text { for all } v \in H \text {, }
$$

where $b(u, v)=(F \nabla u, \nabla v)_{L^{2}(D)}$ and $L(v)=(f, v)_{L^{2}(D)}$. The Lax-Milgram theorem (see e.g. [4] or [14]) ensures (3) has a unique solution if $L(\cdot)$ is a con-

Received February 21, 1995; in revised form December 11, 1995. 
tinuous linear functional on $H$ and the bilinear form, $b(\cdot, \cdot)$, is continuous and coercive on $H \times H$. The purpose of this paper is to extend this argument for elliptic PDEs and similar arguments for parabolic and hyperbolic PDEs to the corresponding SPDEs.

The solution of (1)-(2) found in [6] when $F=\exp ^{\diamond} W_{\phi_{x}}$ and $f, g$ are as above is a function $u: D \rightarrow(\mathscr{S})^{-1}$, where $(\mathscr{S})^{-1}:=\cup_{k=0}^{\infty}(\mathscr{S})^{-1,-k}$ denotes the Kondratiev space (see [11]) and $(\mathscr{S})^{\rho, k}$ denotes the Kondratiev Hilbert spaces defined in the next section. The variational approach requires studying the problem in a Hilbert space. We therefore in Section 2 introduce two families of Hilbert spaces, $(\mathscr{S})^{\rho, k, m}(D)$ and $(\mathscr{S})_{0}^{\rho, k, m}(D)$ isomorphic to $(\mathscr{S})^{\rho, k} \otimes H^{m}(D)$ and $(\mathscr{S})^{\rho, k} \otimes H_{0}^{m}(D)$, respectively. These spaces have the additional advantage that with them we can prove regularity results for the solution in the space variable. This is not possible when one uses the methods developed in [6], [7], [8], [9], and [12]. In Section 2 we also define basic operations on $(\mathscr{S})^{\rho, k, m}(D)$ and $(\mathscr{S})_{0}^{\rho, k, m}(D)$. With these definitions we can, when $g=0$, pass from (1)-(2) to the variational problem (3), with $H=(\mathscr{S})_{0}^{\rho, k, 1}(D), b(u, v)=(F \diamond \nabla u, \nabla v)$, and $L(v)=(f, v)$, where $(\cdot, \cdot)$ denotes the inner product in $(\mathscr{S})^{\rho, k, 0}(D)$. Before we can apply the Lax-Milgram theorem we have to show $b(\cdot, \cdot)$ is continuous and coercive on $H \times H$. Estimates which together with Poincare's inequality give this result for a family of stochastic processes $F$, are obtained in Section 3.

In Section 4, Section 5, and Section 6 we illustrate how variational methods can be used to prove existence and uniqueness of solution for SPDEs of elliptic, parabolic, and hyperbolic type, respectively. Finally, Section 7 contains some concluding remarks.

We remark that an approach related to the one we present below has been suggested by J.-L. Lions and U. Frisch (see [3]). Their idea was to study stochastic differential equations on the direct integral $\int{ }^{\oplus} H(\omega) d \mu(\omega)$, where $H(\omega)$ is a Hilbert space for each $\omega$. The new spaces we introduce may be expressed as $\int{ }^{\oplus} H^{m}(D) d \nu_{\rho, k}(\alpha)$ where $H^{m}(D)$ is the usual Sobolev space over $D \subseteq \mathrm{R}^{d}$ and $\nu_{\rho, k}(\alpha)$ is a counting measure on the set of all multi-indices.

\section{Preliminaries.}

Let $\mathscr{S}:=\mathscr{S}\left(\mathrm{R}^{d}\right)$ denote the Schwartz functions on $\mathrm{R}^{d}$, endowed with the usual Fréchet topology, and let $\mathscr{S}^{\prime}:=\mathscr{S}^{\prime}\left(\mathrm{R}^{d}\right)$ denote its dual endowed with the weak-* topology. Then the Bochner-Minlos theorem ensures the existence of a probability measure $\mu$ on the Borel sets of $\mathscr{S}^{\prime}, B:=B\left(\mathscr{S}^{\prime}\right)$, satisfying

$$
\int_{\mathscr{S}^{\prime}} e^{i\langle\omega, \phi\rangle} d \mu(\omega)=\exp \left(-\frac{1}{2}\|\phi\|^{2}\right) \text { for every } \phi \in \mathscr{S}
$$


where $\|\phi\|^{2}=\int_{\mathrm{R}^{d}} \phi(x)^{2} d x$. The triple $\left(\mathscr{S}^{\prime}, B, \mu\right)$ is called the white noise probability space. Let

$$
h_{i}(x)=(-1)^{i} e^{x^{2} / 2} \frac{d^{i}}{d x^{i}} e^{-x^{2} / 2}, i \in \mathrm{N}_{0}:=\{0,1, \ldots\}
$$

denote the Hermite polynomials and define the Hermite functions by

$$
\xi_{i}(x):=\pi^{-1 / 4}((i-1) !)^{-1 / 2} e^{-x^{2} / 2} h_{i-1}(\sqrt{2} x), i \in \mathrm{N}:=\{1,2, \ldots\} .
$$

Throughout this paper $\left\{e_{i}\right\}_{i=1}^{\infty} \subset \mathscr{S}$ denotes a fixed orthonormal basis for $L^{2}\left(\mathrm{R}^{d}\right)$ defined by taking tensor products of the $\xi_{i}$. That is, if $\beta^{(i)}=\left(\beta_{1}^{(i)}, \beta_{2}^{(i)}, \ldots, \beta_{d}^{(i)}\right)$ is multi-index number $i$ in some fixed ordering of all multi-indices $\beta=\left(\beta_{1}, \ldots, \beta_{d}\right) \in \mathrm{N}^{d}$, we let

$$
e_{i}:=\xi_{\beta_{1}^{(i)}} \otimes \xi_{\beta_{2}^{(i)}} \otimes \cdots \otimes \xi_{\beta_{d}^{(i)}} .
$$

Define

$$
H_{\alpha}(\omega):=\prod_{i=1}^{\infty} h_{\alpha_{i}}\left(\left\langle\omega, e_{i}\right\rangle\right)
$$

for all $\alpha \in I$, where

$$
I:=\left\{\alpha=\left(\alpha_{1}, \alpha_{2}, \ldots\right) \in\left(\mathrm{N}_{0}\right)^{\mathrm{N}}: \alpha_{i}=0 \text { for all but a finite number of } i\right\} .
$$

It is shown in [5] that $\left\{H_{\alpha}: \alpha \in I\right\}$ forms an orthogonal basis for $L^{2}(\mu):=L^{2}\left(\mathscr{S}^{\prime}, B, \mu\right)$, with $E\left[H_{\alpha} H_{\beta}\right]=\delta_{\alpha, \beta} \alpha$ !, where $\alpha !:=\prod_{i=1}^{\infty} \alpha_{i} !$.

The Kondratiev Hilbert spaces are defined as follows. For $-1 \leq \rho \leq 1$ and $k \in \mathrm{R}$ we consider the inner product spaces

$$
(\mathscr{S})^{\rho, k}:=\left\{f=\sum_{\alpha} f_{\alpha} H_{\alpha}: f_{\alpha} \in \mathrm{R} \text { for } \alpha \in I \text { and }\|f\|_{\rho, k}<\infty\right\},
$$

where $\|\cdot\|_{\rho, k}$ is the norm associated with the inner product

$$
(f, g)_{\rho, k}:=\sum_{\alpha} f_{\alpha} g_{\alpha}(\alpha !)^{1+\rho}(2 \mathrm{~N})^{\alpha k} .
$$

In (4) we have used the notation

$$
(2 \mathrm{~N})^{\alpha}:=\prod_{i=1}^{\infty}\left(2^{d} \beta_{1}^{(i)} \beta_{2}^{(i)} \cdots \beta_{d}^{(i)}\right)^{\alpha_{i}}, \alpha=\left(\alpha_{1}, \alpha_{2}, \ldots\right) \in I .
$$

It is not difficult to verify the following proposition:

Proposition 1. For every pair $(\rho, k)$ with $-1 \leq \rho \leq 1$ and $k \in \mathrm{R},(\mathscr{S})^{\rho, k}$ equipped with the inner product (4) is a separable Hilbert space. 
REMARK 1. If $\rho \in[-1,0)$ and $k<0$ an element in $(\mathscr{S})^{\rho, k}$ is a formal sum, in particular the sum $\sum_{\alpha} f_{\alpha} H_{\alpha}$ does not necessarily converge in $L^{1}(\mu)$.

The reason these spaces are not considered for $\rho<-1$ is that in this case it is not possible to define the $\mathscr{S}$-transform (see [11]).

From the definition of $(\mathscr{S})^{\rho, k}$ we see that $(\mathscr{S})^{0,0}=L^{2}(\mu)$. Moreover, if $0 \leq \rho \leq 1$

$$
(\mathscr{S})^{\rho, k} \subset(\mathscr{S})^{\rho, \ell} \subset(\mathscr{S})^{\rho, 0} \subseteq(\mathscr{S})^{-\rho, 0} \subset(\mathscr{S})^{-\rho,-\ell} \subset(\mathscr{S})^{-\rho,-k}
$$

for every $k>\ell>0$ where $(\mathscr{S})^{-\rho,-k}$ is the dual of $(\mathscr{S})^{\rho, k}$ with respect to $L^{2}(\mu)$.

We now introduce a family of Hilbert spaces which turn out to be useful when stochastic partial differential equations are investigated. Fix an open set $D \subseteq \mathrm{R}^{d}$ and let $(\cdot, \cdot)_{m, D}$, or simply $(\cdot, \cdot)_{m}$ if $D$ is clear from the context, denote the usual inner product on the real Sobolev space $H^{m}(D)$ for $m \in \mathrm{N}_{0}$. We then define the inner product

$$
(f, g)_{\rho, k, m, D}:=\sum_{\alpha}\left(f_{\alpha}, g_{\alpha}\right)_{m, D}(\alpha !)^{1+\rho}(2 \mathrm{~N})^{k \alpha}
$$

on the set of functions of the form

$$
f(x)=\sum_{\alpha} f_{\alpha}(x) H_{\alpha}
$$

where $f_{\alpha} \in H^{m}(D)$ for every multi-index $\alpha$.

Definition 1. Let $(\mathscr{S})^{\rho, k, m}(D)\left(\operatorname{resp} .(\mathscr{S})_{0}^{\rho, k, m}(D)\right)$ denote the set

$$
\begin{gathered}
\left\{f(x)=\sum_{\alpha} f_{\alpha}(x) H_{\alpha}: f_{\alpha} \in H^{m}(D) \forall \alpha \in I\left(\text { resp. } H_{0}^{m}(D) \forall \alpha \in I\right),\right. \text { and } \\
\left.\|f\|_{\rho, k, m, D}:=(f, f)_{\rho, k, m, D}^{1 / 2}<\infty\right\}
\end{gathered}
$$

equipped with the inner product (6). We write $(\mathscr{S})^{\rho, k, m},(\mathscr{S})_{0}^{\rho, k, m}$, and $\|\cdot\|_{\rho, k, m}$ if $D$ is clear from the context.

Recall that $H_{0}^{m}(D)$ is defined as the completion of $C_{0}^{\infty}(D)$ with respect to the $\|\cdot\|_{m, D^{-n o r m}}$ and that $L^{2}(D)=H^{0}(D)$. Hence $(\mathscr{S})^{\rho, k, m}$ and $(\mathscr{S})_{0}^{\rho, k, m}$ are equipped with the same norm and $(\mathscr{S})_{0}^{\rho, k, 0}=(\mathscr{S})^{\rho, k, 0}$. The latter simplifies the statements in some of the propositions that ensue. Using that $H^{m}(D)$ is a separable Hilbert space the following result follows from a standard argument.

Proposition 2. If $-1 \leq \rho \leq 1$ and $k \in \mathrm{R}$, then $(\mathscr{S})^{\rho, k, m} \cong(\mathscr{S})^{\rho, k} \otimes H^{m}(D)$ and $(\mathscr{S})_{0}^{\rho, k, m} \cong(\mathscr{S})^{\rho, k} \otimes H_{0}^{m}(D)$ for $m \in \mathrm{N}_{0}$. Moreover, $(\mathscr{S})^{\rho, k, m}$ and $(\mathscr{S})_{0}^{\rho, k, m}$ are separable Hilbert spaces. 
For $m \in \mathrm{N}_{0}$ we recover the inclusions (5)

$$
(\mathscr{S})^{\rho, k, m} \subset(\mathscr{S})^{\rho, \ell, m} \subset(\mathscr{S})^{\rho, 0, m} \subseteq(\mathscr{S})^{-\rho, 0, m} \subset(\mathscr{S})^{-\rho,-\ell, m} \subset(\mathscr{S})^{-\rho,-k, m}
$$

when $\rho \in[0,1]$ and $k>\ell>0$. If $\rho$ and $k$ are fixed, then $(\mathscr{S})^{\rho, k, m_{1}} \subseteq(\mathscr{S})^{\rho, k, m_{2}}$ for $m_{1}, m_{2} \in \mathrm{N}_{0}$ with $m_{1} \geq m_{2}$. Combining these results we see that

$$
(\mathscr{S})^{\rho_{1}, k_{1}, m_{1}} \subseteq(\mathscr{S})^{\rho_{2}, k_{2}, m_{2}}
$$

when $\rho_{1} \geq \rho_{2}, k_{1} \geq k_{2}$, and $m_{1} \geq m_{2}$. Moreover, $(\mathscr{S})^{\rho_{1}, k_{1}, m_{1}}$ is a proper subset of $(\mathscr{S})^{\rho_{2}, k_{2}, m_{2}}$ if and only if one of the inequalities are strict. Similar results for $(\mathscr{S})_{0}^{\rho, k, m}$ are also valid.

In some cases we can say more as the following proposition shows:

Proposition 3. For any $-1 \leq \rho \leq 1, k \in \mathrm{R}, m \in \mathrm{N}$, and open set $D \subseteq \mathrm{R}^{d}$ the canonical embedding

$$
\operatorname{id}_{m}:(\mathscr{S})_{0}^{\rho, k, m} \rightarrow(\mathscr{S})^{\rho, k, 0}
$$

is a continuous injection with dense image.

Proof. Let $i_{m}: H_{0}^{m}(D) \rightarrow L^{2}(D)$ denote the canonical embedding and define

$$
\operatorname{id}_{m}(f):=\sum_{\alpha} i_{m}\left(f_{\alpha}\right) H_{\alpha},
$$

for $f=\sum_{\alpha} f_{\alpha} H_{\alpha} \in(\mathscr{S})_{0}^{\rho, k, m}$. Since $i_{m}$ is a continuous injection with dense image the proposition follows.

The existence of a continuous injection is often expressed compactly as $V \stackrel{\text { id }}{\hookrightarrow} H$, or simply as $V \hookrightarrow H$ if the injection, id, is clear from the context. $\stackrel{\text { id }}{\hookrightarrow}$ means $V$ may be viewed as a subspace of $H$ endowed with a stronger topology.

One of the main reasons for introducing $(\mathscr{S})^{\rho, k, m}$ and $(\mathscr{S})_{0}^{\rho, k, m}$ for $m \geq 1$, is that elements from these spaces have derivatives.

Definition 2. Let $D \subseteq \mathrm{R}^{d}$ be an open set and $m \in \mathrm{N}$. If $\beta \in \mathrm{N}_{0}^{d}$ and $|\beta| \leq m$ we define

$$
\frac{\partial^{\beta}}{\partial x^{\beta}} f:=\sum_{\alpha} \frac{\partial^{\beta} f_{\alpha}}{\partial x^{\beta}} H_{\alpha},
$$

for any $f=\sum_{\alpha} f_{\alpha} H_{\alpha} \in(\mathscr{S})^{\rho, k, m} . \partial^{\beta} f_{\alpha} / \partial x^{\beta}$ is interpreted in the usual $L^{2}(D)$ sense. We shall often write $\partial_{x}^{\beta}$ or $\partial^{\beta}$ for $\partial^{\beta} / \partial x^{\beta}$.

With this definition 


$$
\partial_{x}^{\beta}:(\mathscr{S})^{\rho, k, m} \rightarrow(\mathscr{S})^{\rho, k, m-|\beta|}
$$

is a continuous linear operator, if $|\beta| \leq m$.

Example. For $\phi \in L^{2}\left(\mathrm{R}^{d}\right)$ and $x \in \mathrm{R}^{d}$ we define the smoothed white noise process to be

$$
W_{\phi_{x}}:=\sum_{i=1}^{\infty}\left(\phi_{x}, e_{i}\right)_{0, R^{d}} H_{\varepsilon_{i}}
$$

where $\phi_{x}(\cdot):=\phi(\cdot-x)$ and $\varepsilon_{i}=(0, \ldots, 0,1,0, \ldots)$ denotes the multi-index whose only nonzero entry is a 1 in the $i$ th position. One can show that for every $x \in \mathrm{R}^{d}$ the sum in (8) converges to the usual definition, $\left\langle\omega, \phi_{x}\right\rangle$, in

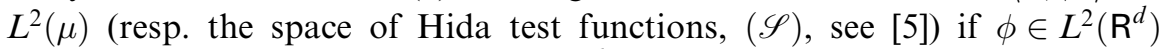
(resp. $\phi \in \mathscr{S}$ ). Moreover, $W_{\phi_{x}} \in(\mathscr{S})^{\rho, k, 0}$ for all $\phi \in L^{2}\left(\mathrm{R}^{d}\right)$ if $-1 \leq \rho \leq 1$, $k \leq 0$, and $D$ is a bounded open subset of $\mathrm{R}^{d}$.

If $\phi \in \mathscr{S}$ and $\left(\phi_{x}, e_{i}\right)_{0, \mathrm{R}^{d}}$ is viewed as the convolution of two Schwartz functions we have that

$$
\partial_{x}^{\beta}\left(\phi_{x}, e_{i}\right)_{0, \mathrm{R}^{d}}=\left(\partial_{x}^{\beta} \phi_{x}, e_{i}\right)_{0, \mathrm{R}^{d}}
$$

for every $\beta \in \mathrm{N}_{0}^{d}$, and Definition 2 implies that

$$
\partial_{x}^{\beta} W_{\phi_{x}}=\sum_{i=1}^{\infty}\left(\partial_{x}^{\beta} \phi_{x}, e_{i}\right)_{0, \mathrm{R}^{d}} H_{\varepsilon_{i}} .
$$

Since $\partial^{\beta} \phi \in \mathscr{S}\left(\mathbf{R}^{d}\right) \subset L^{2}\left(\mathbf{R}^{d}\right)$, summation over all $|\beta| \leq m$ shows $W_{\phi_{x}} \in(\mathscr{S})^{\rho, k, m}$ for all $-1 \leq \rho \leq 1, k \leq 0, m \in \mathrm{N}_{0}$, and bounded open sets $D \subset \mathrm{R}^{d}$.

Suppose $\phi \in \mathscr{S}$ and let $\tilde{\phi}(z):=\phi(-z)$, then $\left\langle\omega, \phi_{x}\right\rangle=\omega * \tilde{\phi}(x)$. Since $\omega * \tilde{\phi}(x)$ is a $C^{\infty}$-function with at most polynomial growth in $x, W_{\phi_{x}}$ cannot in general belong to $(\mathscr{S})^{\rho, k, 0}$ for unbounded sets $D$. For the same reason $W_{\phi_{x}}$ does not generally belong to $(\mathscr{S})_{0}^{\rho, k, 0}$. But by extending the proof of Theorem 2.1 in [15] to $\mathrm{R}^{d}$, we obtain the following lemma whose proof is left to an appendix.

Lemma 1. If $d \geq 1$ and $k>1+\log _{2} d$, then $\sum_{\alpha}(2 \mathrm{~N})^{-k \alpha}<\infty$.

Thus, if $D \subseteq \mathrm{R}^{d}$ is unbounded, $W_{\phi_{x}} \in(\mathscr{S})^{\rho, k, m}$ when $-1 \leq \rho \leq 1$, $k<-1-\log _{2} d$, and $m \in \mathrm{N}_{0}$. Hence the above results can be extended to unbounded open sets $D \subseteq \mathrm{R}^{d}$ by requiring $k<-1-\log _{2} d$. Note also that

$$
\frac{\partial^{\beta}}{\partial x^{\beta}}\left\langle\omega, \phi_{x}\right\rangle=\frac{\partial^{\beta}}{\partial x^{\beta}}(\omega * \tilde{\phi})(x)=\omega *\left(\partial^{\beta} \tilde{\phi}\right)(x)=\left\langle\omega, \partial_{x}^{\beta} \phi_{x}\right\rangle
$$


for every $\omega \in \mathscr{S}^{\prime}$ and $\phi \in \mathscr{S}$. It follows that for the process $W_{\phi_{x}}$ differentiation in the sense of Definition 2 coincides with the usual one.

A closely related process we frequently use in examples is the singular white noise process

$$
W_{x}:=\sum_{i=1}^{\infty} e_{i}(x) H_{\varepsilon_{i}} .
$$

It is easily seen from Lemma 1 that $W_{x} \in(\mathscr{S})^{\rho, k, 0}(D)$ for any open set $D \subseteq \mathrm{R}^{d}$, if $-1 \leq \rho \leq 1$, and $k<-1-\log _{2} d$.

We would also like to compute the expectation of elements in $(\mathscr{S})^{\rho, k, m}$ for general $\rho, k$, and $m \in \mathrm{N}_{0}$. Inspired by [6] we observe that if $f=\sum_{\alpha} f_{\alpha} H_{\alpha} \in(\mathscr{S})^{0,0,0}$, then

$$
E[f]=E[f \cdot 1]=\sum_{\alpha} f_{\alpha} E\left[H_{\alpha} \cdot 1\right]=\sum_{\alpha} f_{\alpha} E\left[H_{\alpha} \cdot H_{(0, \ldots)}\right]=f_{(0, \ldots)} \in L^{2}(D),
$$

using $E\left[H_{\alpha} H_{\beta}\right]=\delta_{\alpha, \beta} \alpha$ !. It is therefore reasonable to define:

Definition 3. The (generalized) expectation of $f=\sum_{\alpha} f_{\alpha} H_{\alpha} \in(\mathscr{S})^{\rho, k, m}$ (resp. $(\mathscr{S})_{0}^{\rho, k, m}$ ) is the deterministic function

$$
E[f]:=f_{(0, \ldots)}: D \rightarrow \mathrm{C},
$$

which belongs to $H^{m}(D)$ (resp. $H_{0}^{m}(D)$.)

We use the term generalized expectation since an $f \in(\mathscr{S})^{\rho, k, m}$ not necessarily is integrable with respect to the measure $\mu$. We conclude this section with the definition of the Wick product.

Definition 4. If $f=\sum_{\alpha} f_{\alpha} H_{\alpha}$ and $g=\sum_{\alpha} g_{\alpha} H_{\alpha}$ are two formal series, we define their Wick product, $f \diamond g$, to be the formal series

$$
f \diamond g:=\sum_{\alpha, \beta} f_{\alpha} g_{\beta} H_{\alpha+\beta}=\sum_{\gamma}\left(\sum_{\alpha+\beta=\gamma} f_{\alpha} g_{\beta}\right) H_{\gamma} .
$$

\section{Two Estimates on the Wick Product.}

Recall that $L^{2}(\mu)$ is not closed under Wick multiplication. To see this let, for example, $f=H_{\varepsilon_{1}}$, then $g \mapsto f \diamond g$ is a densely defined unbounded linear operator on $(\mathscr{S})^{0,0}$. If $f, g \in(\mathscr{S})^{0,0,0}$ we have the additional problem that $f_{\alpha} g_{\beta}$ need not belong to $L^{2}(D)$. To provide conditions on $f$ such that $g \mapsto f \diamond g$ defines a continuous linear operator on $(\mathscr{S})^{-1, k, 0}$ we introduce the Banach spaces $\mathscr{F}_{\ell}$. For open $D \subseteq \mathrm{R}^{d}$ and $\ell \in \mathrm{R}$ we let 


$$
\begin{aligned}
& \mathscr{F}_{\ell}(D):=\left\{f(x)=\sum_{\alpha} f_{\alpha}(x) H_{\alpha}: f_{\alpha} \text { is measurable on } D \text { for every } \alpha\right. \text { and } \\
& \left.\|f\|_{\ell, *}:=\operatorname{ess} \sup _{x \in D}\left(\sum_{\alpha}\left|f_{\alpha}(x)\right|(2 \mathrm{~N})^{\ell \alpha}\right)<\infty\right\} .
\end{aligned}
$$

We suppress the set, $D$, in the notation whenever it is clear from the context.

Proposition 4. Let $D$ be an open subset of $\mathrm{R}^{d}$ and $\ell \in \mathrm{R}$. Then $f \in \mathscr{F}_{\ell}$ defines a continuous linear operator on $(\mathscr{S})^{-1, k, 0}$ by $g \mapsto f \diamond g$ when $k \leq 2 \ell$. Moreover

(9) $\quad\|f \diamond g\|_{-1, k, 0} \leq\|f\|_{k / 2, *}\|g\|_{-1, k, 0} \leq\|f\|_{\ell, *}\|g\|_{-1, k, 0}$ for $g \in(\mathscr{S})^{-1, k, 0}$.

Proof. It suffices to prove (9). Suppose $f=\sum_{\alpha} f_{\alpha} H_{\alpha} \in \mathscr{F}_{\ell}$, $g=\sum_{\beta} g_{\beta} H_{\beta} \in(\mathscr{S})^{-1, k, 0}$, and $k \leq 2 \ell$, then Lebesgue's monotone convergence theorem implies

$$
\|f \diamond g\|_{-1, k, 0}^{2}=\int_{D} \sum_{\gamma}\left(\sum_{\alpha+\beta=\gamma} f_{\alpha} g_{\beta}\right)^{2}(2 \mathrm{~N})^{k \gamma} d x
$$

For $n \in \mathrm{N}$ let $I_{n}:=\left\{\left(\alpha_{1}, \alpha_{2}, \ldots\right) \in I: \alpha_{j}=0\right.$ for $\left.j>n\right\}$. If $n \in \mathrm{N}, x \in D$, and $\tilde{\alpha} \in \mathrm{Z}^{n}$ we define

$$
\tilde{f}_{x}^{(n)}(\tilde{\alpha}):= \begin{cases}f_{\alpha}(x)(2 \mathrm{~N})^{k \alpha / 2} & \text { if } \alpha=\left(\tilde{\alpha}_{1}, \ldots, \tilde{\alpha}_{n}, 0, \ldots\right) \in I_{n} . \\ 0 & \text { otherwise }\end{cases}
$$

Suppose $\tilde{g}_{x}^{(n)}(\tilde{\alpha})$ is defined similarly, then $\left\{\tilde{f}_{x}^{(n)}(\tilde{\alpha})\right\}_{\tilde{\alpha} \in Z^{n}} \in \ell^{1}\left(Z^{n}\right)$ and $\left\{\tilde{g}_{x}^{(n)}(\tilde{\alpha})\right\}_{\tilde{\alpha} \in Z^{n}} \in \ell^{2}\left(Z^{n}\right)$ for almost every $x \in D$. Hence

$$
\sum_{\gamma \in I_{n}}\left(\sum_{\alpha+\beta=\gamma} f_{\alpha} g_{\beta}\right)^{2}(2 \mathrm{~N})^{k \gamma}=\sum_{\tilde{\gamma} \in Z^{n}}\left(\sum_{\tilde{\alpha} \in Z^{n}} \tilde{f}_{x}^{(n)}(\tilde{\alpha}) \tilde{g}_{x}^{(n)}(\tilde{\gamma}-\tilde{\alpha})\right)^{2}=\left\|\tilde{f}_{x}^{(n)} *_{n} \tilde{g}_{x}^{(n)}\right\|_{\ell^{2}\left(Z^{n}\right)}^{2}
$$

for a.e. $x \in D$, where $*_{n}$ denotes the convolution on $\mathrm{Z}^{n}$ with respect to counting measure. Young's inequality provides the upper bound

$$
\left\|\tilde{f}_{x}^{(n)} *_{n} \tilde{g}_{x}^{(n)}\right\|_{\ell^{2}\left(Z^{n}\right)} \leq\left\|\tilde{f}_{x}^{(n)}\right\|_{\ell^{1}\left(Z^{n}\right)}\left\|\tilde{g}_{x}^{(n)}\right\|_{\ell^{2}\left(Z^{n}\right)} \text { a.e. } x \in D
$$

for every $n \in \mathrm{N}$. Therefore

$$
\|f \diamond g\|_{-1, k, 0} \leq\left[\int_{D} \lim _{n \rightarrow \infty}\left\|\tilde{f}_{x}^{(n)}\right\|_{\ell^{1}\left(\mathbf{Z}^{n}\right)}^{2}\left\|\tilde{g}_{x}^{(n)}\right\|_{\ell^{2}\left(\mathbf{Z}^{n}\right)}^{2} d x\right]^{1 / 2} \leq\|f\|_{k / 2, *}\|g\|_{-1, k, 0}
$$

and since $k / 2 \leq \ell$ we have $\|f\|_{k / 2, *} \leq\|f\|_{\ell, *}$, which completes the proof. 
A corresponding result for ordinary Kondratiev Hilbert spaces, $(\mathscr{S})^{-1, k}$, with no $x$ dependence becomes a corollary.

Corollary 1. If $f=\sum_{\alpha} f_{\alpha} H_{\alpha}$ is such that $s:=\sum_{\alpha}\left|f_{\alpha}\right|(2 \mathrm{~N})^{k \alpha / 2}<\infty$, then $g \mapsto f \diamond g$ defines a continuous linear operator on $(\mathscr{S})^{-1, k}$ with norm less than or equal to $s$. Moreover, if $\ell>k+1+\log _{2} d$, then any $f \in(\mathscr{S})^{-1, \ell}$ defines a continuous linear operator on $(\mathscr{S})^{-1, k}$ by $g \mapsto f \diamond g$ such that

$$
\|f \diamond g\|_{-1, k} \leq\left(\sum_{\alpha}(2 \mathrm{~N})^{(k-\ell) \alpha}\right)^{1 / 2}\|f\|_{-1, \ell}\|g\|_{-1, k}<\infty \text { for } g \in(\mathscr{S})^{-1, k}
$$

Proof. The first part follows from Proposition 4. To prove the second, note that

$$
\begin{aligned}
s=\sum_{\alpha}\left|f_{\alpha}\right|(2 \mathrm{~N})^{k \alpha / 2} & =\sum_{\alpha}\left(\left|f_{\alpha}\right|(2 \mathrm{~N})^{\ell / 2}\right) \cdot(2 \mathrm{~N})^{(k-\ell) \alpha / 2} \\
& \leq\|f\|_{-1, \ell}\left(\sum_{\alpha}(2 \mathrm{~N})^{(k-\ell) \alpha}\right)^{1 / 2} .
\end{aligned}
$$

The statement now follows from Lemma 1.

Corollary 1 generalizes results as Corollary 4.22 in [5]. It seems impossible to extend the corollary to Kondratiev spaces with $\rho>-1$ since there is noconstant $K$ such that $(m+n) ! \leq K m ! n$ ! for all $m, n \in \mathrm{N}$.

$W_{\phi_{x}}$ belongs to $\mathscr{F}_{\ell}(D)$ for arbitrary $\phi \in L^{2}\left(\mathrm{R}^{d}\right)$, open set $D \subseteq \mathrm{R}^{d}$, and $\ell<-\left(1+\log _{2} d\right) / 2$. To see this note that by Schwarz' inequality

$$
\left\|W_{\phi_{x}}\right\|_{\ell, *}=\operatorname{ess} \sup _{x \in D} \sum_{i=1}^{\infty}\left|\left(\phi_{x}, e_{i}\right)_{0, \mathrm{R}^{d}}\right|(2 \mathrm{~N})^{\ell \varepsilon_{i}} \leq\|\phi\|_{0, \mathrm{R}^{d}}\left(\sum_{\alpha}(2 \mathrm{~N})^{2 \ell \alpha}\right)^{1 / 2},
$$

which by Lemma 1 is finite if $2 \ell<-1-\log _{2} d$. Using 22.14 .17 in [1] to obtain $\left|e_{i}(x)\right| \leq\left(C \pi^{-1 / 4}\right)^{d}$ for $x \in \mathrm{R}^{d}$ and $i \in \mathrm{N}$, where $C \approx 1.086435$, it can be shown that the singular white noise $W_{x} \in \mathscr{F}_{\ell}$ if $\ell<-1-\log _{2} d$.

When we turn to consider SPDEs it will be important to be able to determine when the bilinear form $b\left(g_{1}, g_{2}\right)=\left(f \diamond g_{1}, g_{2}\right)_{-1, k, 0}$ is coercive, that is, if there exists a $C>0$ such that $b(g, g) \geq C\|g\|_{-1, k, 0}^{2}$ for every $g \in(\mathscr{S})^{-1, k, 0}$. Let

$$
\begin{aligned}
\mathscr{P}_{\ell}(D):=\left\{f \in \mathscr{F}_{\ell}(D):\right. & \exists A>0 \text { such that } \\
& \left.\left(f_{(0, \ldots)} g, g\right)_{0, D} \geq A\|g\|_{0, D}^{2} \forall g \in L^{2}(D)\right\} .
\end{aligned}
$$

Note that $f \in \mathscr{F}_{\ell}$ ensures $b(\cdot, \cdot)$ is continuous on $(\mathscr{S})^{-1, k, 0}$ for $k \leq 2 \ell$. The second condition is necessary, otherwise $b(\cdot, \cdot)$ would fail to be coercive on 
the subspace $\left\{g H_{(0, \ldots)}: g \in L^{2}(D)\right\}$ of $(\mathscr{S})^{-1, k, 0}$. The following proposition shows the conditions are also sufficient, provided $k$ is small enough.

Proposition 5. Let $D \subseteq \mathrm{R}^{d}$ be open and $f \in \mathscr{P}_{\ell}(D)$ for some real $\ell$. Then there exist constants $K=K(f) \leq 2 \ell$ and $C=C(f)>0$ such that

$$
(f \diamond g, g)_{-1, k, 0} \geq C\|g\|_{-1, k, 0}^{2} \text { for every } g \in(\mathscr{S})^{-1, k, 0},
$$

when $k<K$.

Proof. Fix $\ell \in \mathrm{R}$ and $f=\sum_{\alpha} f_{\alpha} H_{\alpha} \in \mathscr{P}_{\ell}(D)$, then for $k \leq 2 \ell$

$$
\begin{aligned}
\underset{x \in D}{\operatorname{ess} \sup _{|\alpha|>0}} \sum_{\mid \alpha}\left|f_{\alpha}(x)\right|(2 \mathbf{N})^{k \alpha / 2} & =\underset{x \in D}{\operatorname{ess}} \sup _{|\alpha|>0}\left|f_{\alpha}(x)\right|(2 \mathbf{N})^{\ell \alpha}(2 \mathrm{~N})^{(k / 2-\ell) \alpha} \\
& \leq\|f\|_{\ell, *} 2^{d(k / 2-\ell)} \downarrow 0
\end{aligned}
$$

as $k \downarrow-\infty$. For any $g=\sum_{\beta} g_{\beta} H_{\beta} \in(\mathscr{S})^{-1, k, 0}$ we have

$$
\begin{aligned}
& (f \diamond g, g)_{-1, k, 0}=\sum_{\gamma} \int_{D}\left(\sum_{\alpha+\beta=\gamma} f_{\alpha} g_{\beta}\right) g_{\gamma} d x(2 \mathrm{~N})^{k \gamma} \\
\geq & \sum_{\gamma}\left[\int_{D} f_{(0, \ldots)} g_{\gamma}^{2} d x(2 \mathrm{~N})^{k \gamma}\right. \\
- & \sum_{\alpha+\beta=\gamma,|\alpha|>0} \int_{D}\left(\sqrt{\left|f_{\alpha}\right|(2 \mathrm{~N})^{k \alpha / 2}}\left|g_{\gamma}\right|(2 \mathrm{~N})^{k \gamma / 2}\right) . \\
& \left.\left(\sqrt{\left|f_{\alpha}\right|(2 \mathrm{~N})^{k \alpha / 2}}\left|g_{\beta}\right|(2 \mathrm{~N})^{k \beta / 2}\right) d x\right] .
\end{aligned}
$$

We apply the inequality $a b \leq a^{2} / 2+b^{2} / 2$ to the parentheses under the last integral sign and consider the two resulting terms separately. From (11) the first term is bounded by

$$
\frac{1}{2} \sum_{|\gamma|>0} \int_{D}\left(\sum_{|\alpha|>0}\left|f_{\alpha}(x)\right|(2 \mathrm{~N})^{k \alpha / 2}\right) g_{\gamma}(x)^{2} d x(2 \mathrm{~N})^{k \gamma} \leq \frac{1}{2} 2^{d(k / 2-\ell)}\|f\|_{\ell, *}\|g\|_{-1, k, 0}^{2} .
$$

The second term takes the form 


$$
\begin{aligned}
& \frac{1}{2} \sum_{\gamma} \sum_{\alpha+\beta=\gamma,|\alpha|>0} \int_{D}\left|f_{\alpha}(x)\right|(2 \mathrm{~N})^{k \alpha / 2} g_{\beta}(x)^{2} d x(2 \mathrm{~N})^{k \beta} \\
\leq & \frac{1}{2} \sum_{\beta} \int_{D}\left(\underset{x \in D}{\operatorname{ess} \sup _{|\alpha|>0}} \sum_{|\alpha|}\left|f_{\alpha}(x)\right|(2 \mathrm{~N})^{k \alpha / 2}\right) g_{\beta}(x)^{2} d x(2 \mathrm{~N})^{k \beta} \\
\leq & \frac{1}{2} 2^{d(k / 2-\ell)}\|f\|_{\ell, *}\|g\|_{-1, k, 0}^{2} .
\end{aligned}
$$

From (10), (11), and (12) it follows that if $k<K(f):=2 \ell+$ $2 \log \left(A /\|f\|_{\ell, *}\right) / \log 2^{d} \leq 2 \ell$, then

$$
(f \diamond g, g)_{-1, k, 0} \geq C\|g\|_{-1, k, 0}^{2} \text { for } g \in(\mathscr{S})^{-1, k, 0},
$$

where $C=C(f)=A-2^{d(k / 2-\ell)}\|f\|_{\ell, *}>0$.

A useful result for what follows is:

Proposition 6. Let $D \subseteq \mathrm{R}^{d}$ be open and $\ell \in \mathrm{R}$.

(i) If $f, g \in \mathscr{F}_{\ell}(D)$, then $\|f \diamond g\|_{\ell, *} \leq\|f\|_{\ell, *}\|g\|_{\ell, *}$.

(ii) Suppose $G(x)=\sum_{n=0}^{\infty} c_{n} x^{n}$ is analytic on the open interval $(-R, R)$ for some $R>0$. If $f \in \mathscr{F}_{\ell}(D)$ with $\|f\|_{\ell, *}<R$, then

$$
G^{\diamond}(f):=\sum_{n=0}^{\infty} c_{n} f^{\diamond n} \in \mathscr{F}_{\ell}(D)
$$

and

$$
\left\|G^{\diamond}(f)\right\|_{\ell, *} \leq \sum_{n=0}^{\infty}\left|c_{n}\right|\|f\|_{\ell, *}^{n}<\infty .
$$

(iii) If $f \in \mathscr{F}_{\ell}(D)$, then $\exp ^{\diamond} f:=\sum_{n=0}^{\infty} f^{\diamond n} / n ! \in \mathscr{P}_{\ell}(D)$.

Proof. Suppose $D \subseteq \mathrm{R}^{d}$ is open and $\ell \in \mathrm{R}$. If $f=\sum_{\alpha} f_{\alpha} H_{\alpha}$, $g=\sum_{\alpha} g_{\alpha} H_{\alpha} \in \mathscr{F}_{\ell}(D)$, then

$$
\begin{aligned}
& \|f \diamond g\|_{\ell, *}=\operatorname{ess} \sup _{x \in D} \sum_{\gamma}\left|\sum_{\alpha+\beta=\gamma} f_{\alpha}(x) g_{\beta}(x)\right|(2 \mathrm{~N})^{\ell \gamma} \\
& \leq \operatorname{ess} \sup _{x \in D} \sum_{\alpha} \sum_{\beta}\left|f_{\alpha}(x)\right|(2 \mathrm{~N})^{\ell \alpha}\left|g_{\beta}(x)\right|(2 \mathrm{~N})^{\ell \beta} \leq\|f\|_{\ell, *}\|g\|_{\ell, *} .
\end{aligned}
$$

To prove (ii), note that the triangle inequality together with (i) gives

$$
\left\|\sum_{n=0}^{N} c_{n} f^{\diamond n}\right\|_{\ell, *} \leq \sum_{n=0}^{N}\left|c_{n}\right|\left\|f^{\diamond n}\right\|_{\ell, *} \leq \sum_{n=0}^{N}\left|c_{n}\right|\|f\|_{\ell, *}^{n} \leq \sum_{n=0}^{\infty}\left|c_{n}\right|\|f\|_{\ell, *}^{n} .
$$


Since $G(x)$ is absolutely convergent on compact subsets of $(-R, R)$, it follows that $\sum_{n=0}^{N} c_{n} f^{\diamond n}$ is a Cauchy sequence in $\mathscr{F}_{\ell}$ whose limit, $G^{\diamond}(f)$, satisfies (13).

(iii) follows from (ii) and that the 0th term in the formal expansion for $\exp ^{\diamond} f$ is 1 .

REMARK 2. Similar results are readily obtained for the ordinary Kondratiev spaces $(\mathscr{S})^{-1, k}$.

By Proposition 6, $\exp ^{\diamond} W_{x} \in \mathscr{F}_{\ell}(D)$ for arbitrary $\ell<-1-\log _{2} d$ and open sets $D \subseteq \mathrm{R}^{d}$. Therefore $g \mapsto \exp ^{\diamond} W_{x} \diamond g$ is a continuous linear operator on $(\mathscr{S})^{\rho, k, 0}$ if $\rho=-1$ and $k \leq 2 \ell$. It is in fact possible to show that $\rho=-1$ also is a necessary condition for $g \mapsto \exp ^{\diamond} W_{x} \diamond g$ to be a continuous linear transformation on $(\mathscr{S})^{\rho, k, 0}$.

\section{Elliptic SPDEs.}

In [6] the authors found an explicit solution of the smoothed pressure equation in a stochastic medium. We discuss this equation in detail to illustrate how our ideas can be used to prove an elliptic SPDE has a unique variational solution. The main problem is to determine a suitable Hilbert space on which the bilinear form associated with the equation satisfies the conditions in the Lax-Milgram theorem. When we have found a suitable Hilbert space, which depends on the problem, the Lax-Milgram theorem implies the problem has a unique variational solution.

ExAmple. Let $D \subset \mathrm{R}^{d}$ be an open set of finite width, that is, assume $D$ lies between two parallel hyperplanes. Fix $F \in \mathscr{P}_{\ell}(D)$ for some real $\ell$ and consider

$$
\begin{aligned}
-\nabla \cdot(F \diamond \nabla u) & =f \text { in } D \times \mathscr{S}^{\prime}, \\
\left.u\right|_{\partial D} & =\left.g\right|_{\partial D},
\end{aligned}
$$

where $f \in(\mathscr{S})^{\rho, k, 0}$ and $g \in(\mathscr{S})^{\rho, k, 1}$ are given. We now intend to find a variational formulation in the Hilbert space $(\mathscr{S})^{\rho, k, 1}$. Suppose a solution $u \in(\mathscr{S})^{\rho, k, 1}$ of (14)-(15) for a suitable pair $(\rho, k)$ is known, then

$$
(-\nabla \cdot(F \diamond \nabla u), v)_{\rho, k, 0}=(f, v)_{\rho, k, 0}
$$

for every test function $v \in(\mathscr{S})_{0}^{\rho, k, 1}$. Using the definition of $(\cdot, \cdot)_{\rho, k, 0}$ and integrating each term by parts, we obtain the bilinear form

$$
b_{\rho, k}(u, v):=(F \diamond \nabla u, \nabla v)_{\rho, k, 0}
$$

for the left hand side of (16). 
For given $f \in(\mathscr{S})^{\rho, k, 0}$ and $g \in(\mathscr{S})^{\rho, k, 1}$, the variational formulation of (14)-(15) becomes: Find $u \in(\mathscr{S})^{\rho, k, 1}$ such that

(i) $u-g \in(\mathscr{S})_{0}^{\rho, k, 1}$, and

(ii) $b_{\rho, k}(u, v)=(f, v)_{\rho, k, 0}$ for every $v \in(\mathscr{S})_{0}^{\rho, k, 1}$.

For simplicity we interpret the boundary condition in the generalized sense, (i).

Existence and uniqueness of a variational solution will follow from the Lax-Milgram theorem if $(\rho, k)$ is chosen such that $b_{\rho, k}(u, v)$ is continuous on $(\mathscr{S})^{\rho, k, 1} \times(\mathscr{S})^{\rho, k, 1}$ and coercive on $(\mathscr{S})_{0}^{\rho, k, 1} \times(\mathscr{S})_{0}^{\rho, k, 1}$. If $g=0$, it suffices to assume $b_{\rho, k}(\cdot, \cdot)$ is continuous on $(\mathscr{S})_{0}^{\rho, k, 1} \times(\mathscr{S})_{0}^{\rho, k, 1}$.

Schwarz' inequality shows

$$
\left|b_{\rho, k}(u, v)\right|=\left|(F \diamond \nabla u, \nabla v)_{\rho, k, 0}\right| \leq\|F \diamond \nabla u\|_{\rho, k, 0}\|\nabla v\|_{\rho, k, 0} .
$$

From Proposition 4 and the definition of $\|\cdot\|_{\rho, k, 1}$ we conclude that

$$
\left|b_{-1, k}(u, v)\right| \leq\|F\|_{k / 2, *}\|u\|_{-1, k, 1}\|v\|_{-1, k, 1},
$$

for every $u, v \in(\mathscr{S})^{-1, k, 1}$, when $k \leq 2 \ell$. Thus $b_{-1, k}(\cdot, \cdot)$ is a continuous bilinear form on $(\mathscr{S})^{-1, k, 1} \times(\mathscr{S})^{-1, k, 1}$ if $k \leq 2 \ell$. By Proposition 5 there exist constants $K(F) \leq 2 \ell$ and $C=C(F)>0$ such that

$$
b_{-1, k}(u, u) \geq C\|\nabla u\|_{-1, k, 0}^{2}, \text { for all } u \in(\mathscr{S})_{0}^{-1, k, 1},
$$

if $k<K(F)$. Since $D$ is assumed to have finite width, Poincaré's inequality implies

$$
b_{-1, k}(u, u) \geq C \tilde{C}\|u\|_{-1, k, 1}^{2}, \tilde{C}=\tilde{C}(D)>0,
$$

for every $u \in(\mathscr{S})_{0}^{-1, k, 1}$ when $k<K(F)$. Thus, if $k<K(F)$ then $b_{-1, k}(\cdot, \cdot)$ satisfies the conditions in the Lax-Milgram theorem and (14)-(15) has the unique variational solution $u=h+g$ where $h \in(\mathscr{S})_{0}^{-1, k, 1}$ satisfies

$$
b_{-1, k}(h, v)=(f, v)_{-1, k, 0}-b_{-1, k}(g, v) \text { for every } v \in(\mathscr{S})_{0}^{-1, k, 1} .
$$

The following theorem is an almost immediate consequence of the example.

THEOREM 1. Let $D \subset \mathrm{R}^{d}$ be an open set of finite width and suppose $F \in \mathscr{P}_{\ell}(D)$ for some real $\ell$. Then there exists a constant $K(F) \leq 2 \ell$ such that if $k<K(F)$, (14)-(15) has a unique variational solution $u \in(\mathscr{S})^{-1, k, 1}$ for every $f \in(\mathscr{S})^{-1, k, 0}$ and $g \in(\mathscr{S})^{-1, k, 1}$.

Moreover, $E[u]$ is the variational solution of the deterministic problem that results from (14)-(15) when $F, f$, and $g$ are replaced by $E[F], E[f]$, and $E[g]$, respectively. 
Proof. It only remains to prove the last statement. Let $F=\sum_{\alpha} F_{\alpha} H_{\alpha}$ and suppose $u=\sum_{\alpha} u_{\alpha} H_{\alpha}$ is the variational solution of (14)-(15) for given $f \in(\mathscr{S})^{-1, k, 0}$ and $g \in(\mathscr{S})^{-1, k, 1}$. Then $u-g \in(\mathscr{S})_{0}^{-1, k, 1}$, which implies

$$
E[u]-E[g]=u_{(0, \ldots)}-g_{(0, \ldots)} \in H_{0}^{1}(D) .
$$

Substituting test functions of the form $v=w H_{(0, \ldots)}$ into the variational formulation of (14)-(15), we obtain

$$
\left(F_{(0, \ldots)} \nabla u_{(0, \ldots)}, \nabla w\right)_{0, D}=\left(f_{(0, \ldots)}, w\right)_{0, D} \text { for all } w \in H_{0}^{1}(D) .
$$

Since $E[F]:=F_{(0, \ldots)}, E[f]=f_{(0, \ldots)}$, and $E[u]=u_{(0, \ldots)}$, this relation together with (17) proves the theorem.

Example. Let $D \subset \mathrm{R}^{d}$ be open and of finite width, fix $F \in \mathscr{P}_{\ell}(D)$ for some real $\ell$, and consider the stochastic Schrödinger equation

$$
\begin{aligned}
-\Delta u+F \diamond u & =f \text { in } D \times \mathscr{S}^{\prime}, \\
\left.u\right|_{\partial D} & =\left.g\right|_{\partial D},
\end{aligned}
$$

for given $f \in(\mathscr{S})^{\rho, k, 0}$ and $g \in(\mathscr{S})^{\rho, k, 1}$. An analysis similar to the one presented in the previous example, leads to the same variational formulation but this time with respect to the bilinear form

$$
b_{\rho, k}(u, v):=(\nabla u, \nabla v)_{\rho, k, 0}+(F \diamond u, v)_{\rho, k, 0} .
$$

Furthermore, one can show Theorem 1 applies verbatim to (18)-(19).

Having proved that there exists a unique variational solution to (18)-(19) for arbitrary $F \in \mathscr{P}_{\ell}(D)$, we next investigate the problem for a particular $F$ with $-F \in \mathscr{P}_{\ell}(D)$. Let $\varepsilon(D)$ denote the Poincaré constant, i.e., the largest positive number such that

$$
\int_{D}|\nabla u|^{2} d x \geq \varepsilon(D) \int_{D}|u|^{2} d x \text { for all } u \in H_{0}^{1}(D) .
$$

If $D$ is bounded, $\varepsilon(D)$ is the smallest eigenvalue of $-\Delta_{D}$. We consider (18)(19) with respect to $F_{\varepsilon}=-\varepsilon \exp ^{\diamond} W_{x}$ for $\varepsilon>0$. In [8] this problem was solved explicitly in $L^{1}(\mu)$ on bounded domains for any $\varepsilon$ with $0<\varepsilon<\varepsilon(D)$. The authors considered the smoothed problem, but our analysis applies to this problem as well. We now show how these results can be recovered in our setting. $b_{-1, k}^{\varepsilon}(\cdot, \cdot)$ is continuous on $(\mathscr{S})^{-1, k, 1} \times(\mathscr{S})^{-1, k, 1}$ when $0<\varepsilon<\infty$ and $k<-2-2 \log _{2} d$ since $F_{\varepsilon} \in \mathscr{F}_{\ell}$ for $\ell<-1-\log _{2} d$. To show $b_{-1, k}^{\varepsilon}(\cdot, \cdot)$ is coercive on $(\mathscr{S})_{0}^{-1, k, 1} \times(\mathscr{S})_{0}^{-1, k, 1}$ for suitably chosen $k$, fix $0<\varepsilon<\varepsilon(D)$ and note that by Proposition 4 and (20) 


$$
\begin{aligned}
b_{-1, k}^{\varepsilon}(u, u)= & (\delta+(1-\delta))\|\nabla u\|_{-1, k, 0}+\left(F_{\varepsilon} \diamond u, u\right)_{-1, k, 0} \\
\geq & \delta\|\nabla u\|_{-1, k, 0}+((1-\delta) \varepsilon(D) \\
& \left.-\left\|F_{\varepsilon}\right\|_{k / 2, *}\right)\|u\|_{-1, k, 0}^{2} \text { for all } u \in(\mathscr{S})_{0}^{-1, k, 1},
\end{aligned}
$$

where $k<-2-2 \log _{2} d$ and $0<\delta<1$. Since $\left\|F_{\varepsilon}\right\|_{k / 2, *} \downarrow \varepsilon$ as $k \downarrow-\infty$, one can choose $k<-2-2 \log _{2} d$ and $0<\delta<1$ such that $b_{-1, k}^{\varepsilon}(\cdot, \cdot)$ is seen to be coercive on $(\mathscr{S})_{0}^{-1, k, 1} \times(\mathscr{S})_{0}^{-1, k, 1}$.

Observe that we cannot invoke the Fredholm alternative to solve (18)-(19) when $\varepsilon \geq \varepsilon(D)$, since the injection $\mathrm{id}_{1}:(\mathscr{S})_{0}^{-1, k, 1} \rightarrow(\mathscr{S})^{-1, k, 0}$ is not compact for any open set $D \subseteq \mathrm{R}^{d}$.

We summarize our results in the following theorem.

THEOREM 2. Let $D \subset \mathrm{R}^{d}$ have finite width, $F_{\varepsilon}=-\varepsilon \exp ^{\diamond} W_{x}$, and $\varepsilon(D)$ be the Poincaré constant. Then for any $0<\varepsilon<\varepsilon(D)$, there exists a $k<-2-2 \log _{2} d$ such that (18)-(19) has a unique variational solution in $(\mathscr{S})^{-1, k, 1}$ for any $f \in(\mathscr{S})^{-1, k, 0}$ and $g \in(\mathscr{S})^{-1, k, 1}$.

To illustrate that our approach does not only apply to second order problems we include the stochastic biharmonic equation

$$
\begin{gathered}
\Delta(F \diamond \Delta u)=f \text { in } D \times \mathscr{S}^{\prime}, \\
\left.u\right|_{\partial D}=\left.g\right|_{\partial D} .
\end{gathered}
$$

Essentially the same argument as for the pressure equation in a stochastic medium gives the following theorem:

THEOREM 3. Let $D \subset \mathrm{R}^{d}$ be an open set of finite width and suppose $F \in \mathscr{P}_{\ell}(D)$ for some real $\ell$. Then there exists a constant $K(F) \leq 2 \ell$ such that if $k<K(F)$, (21)-(22) has a unique variational solution $u \in(\mathscr{S})^{-1, k, 2}$ for every $f \in(\mathscr{S})^{-1, k, 0}$ and $g \in(\mathscr{S})^{-1, k, 2}$.

Moreover, $E[u]$ is the variational solution of the deterministic problem that results from (21)-(22) when $F, f$, and $g$ are replaced by $E[F], E[f]$, and $E[g]$, respectively.

Proposition 6 provides many examples of $F \in \mathscr{P}_{\ell}$ we could use in the preceding examples. The case when $F$ is the smoothed exponential white noise, $F_{\phi}=\exp ^{\diamond} W_{\phi_{x}}$ for $\phi \in \mathscr{S}$, is of particular interest. In [6], the authors find an explicit solution, $u_{S}: D \rightarrow(\mathscr{S})^{-1}$, of (14)-(15) when $F=F_{\phi}, D$ is a bounded open subset of $\mathrm{R}^{d}, f$ is deterministic and Hölder continuous on $\bar{D}$, and $g=0$. The Hermite transform of their solution, $\tilde{u}_{S}(x, z)$, is given by a FeynmanKac formula and satisfies the Hermite transformed equation

$$
-\nabla_{x} \cdot\left(\tilde{F}_{\phi}(x, z) \nabla_{x} \tilde{u}(x, z)\right)=f \text { for } x \in D,\left.\tilde{u}(x, z)\right|_{\partial D}=0 ;
$$


for every $z \in B$. Here $\tilde{F}_{\phi}$ denotes the Hermite transform of $F_{\phi}$ and $B$ is a neighborhood of the origin in the Hermite transform's domain of definition. We refer to [6] and [13] for discussions of the Hermite transform on Kondratiev spaces. Let $u_{V}$ denote the variational solution of (14)-(15) with $F, D$, $f$, and $g$ as above. It is possible to show the Hermite transform of $u_{V}$, $\tilde{u}_{V}(x, z)$, is a variational solution of (23) for every $z \in B$. In general, it is difficult to compare $\tilde{u}_{S}$ and $\tilde{u}_{V}$ since they satisfy (23) with respect to two different generalized solution concepts. But under additional regularity assumptions on $f$ and $D$, standard regularity results may be used to conclude that there is a set $N \subset D$ of Lebesgue measure 0 such that $\tilde{u}_{S}=\tilde{u}_{V}$ for all $x \in D-N$ and $z \in B$. By taking the inverse Hermite transform it follows that $u_{S}=u_{V}$ in $(\mathscr{S})^{-1}$ for a.e. $x \in D$.

\section{Parabolic SPDEs.}

In this section we apply variational methods to prove existence and uniqueness of solutions for SPDEs of first order in $t$. We illustrate our ideas on the following initial boundary value problem. Suppose $0<T<\infty, D \subset \mathrm{R}^{d}$ is an open set of finite width and $F \in \mathscr{P}_{\ell}(D)$ for some real $\ell$. We consider the problem

$$
\begin{aligned}
\frac{d u}{d t}-\nabla_{x} \cdot\left(F \diamond \nabla_{x} u\right) & =f \text { in } D \times(0, T) \times \mathscr{S}^{\prime}, \\
\left.u(t)\right|_{\partial D} & =0 \text { for } 0<t<T, \text { and } \\
u(0) & =u_{0},
\end{aligned}
$$

where $f$ and $u_{0}$ are given stochastic processes. In which sense $u$ satisfies the equations and from which spaces $f$ and $u_{0}$ are chosen, will be made precise shortly. But first we recall some basic definitions and results for variational problems of this type.

Fix $-1 \leq \rho \leq 1$ and $k \in \mathrm{R}$. Let $V$ and $H$ denote the separable Hilbert spaces $(\mathscr{S})_{0}^{\rho, k, 1}(D)$ and $(\mathscr{S})^{\rho, k, 0}(D)$, respectively. By Proposition 3 the continuous injection id $:=\mathrm{id}_{1}: V \rightarrow H$ has a dense image. We identify $H$ with its dual $H^{\prime}$ and define the transpose of id, id $: H^{\prime} \rightarrow V^{\prime}$, by

$$
\left\langle h^{\prime}, \text { id } v\right\rangle_{H}=\left\langle\operatorname{id}^{\prime} h^{\prime}, v\right\rangle_{V},
$$

where $\langle\cdot, \cdot\rangle$ denotes the duality bracket and the subscript indicates the spaces involved. It follows that id' is a continuous injection with a dense image and by definition

$$
V \stackrel{\mathrm{id}}{\hookrightarrow} H \stackrel{\mathrm{id}^{\prime}}{\hookrightarrow} V^{\prime}
$$


is a Gel'fand triple. Since the injections are continuous and have dense images

$$
\left\langle v^{\prime}, v\right\rangle_{V}=\lim _{\operatorname{id}^{\prime} h \rightarrow v^{\prime}}(h, \text { id } v)_{H} \text { for } v \in V, v^{\prime} \in V^{\prime} .
$$

Therefore the inner product on $H$ has a continuous extension to $V^{\prime} \times V$ and we may write $(\cdot, \cdot)_{H}$ for duality bracket $\langle\cdot, \cdot\rangle_{V}$.

Let the space of distributions with values in the Hilbert space $V$, $\mathscr{D}^{\prime}(0, T ; V)$, consist of all continuous linear mappings from $\mathscr{D}(0, T)$ into $V$. If $S \in \mathscr{D}^{\prime}(0, T ; V)$, we define the distributional derivative of $S$ to be the linear mapping

$$
\frac{d S}{d t}(\phi)=-S\left(\frac{d \phi}{d t}\right) \text { for all } \phi \in \mathscr{D}(0, T) .
$$

$\mathscr{D}^{\prime}\left(0, T ; V^{\prime}\right)$ is defined similarly. With these definitions it is possible to prove (see [4]) that

$$
W(0, T):=\left\{v \in L^{2}(0, T ; V): \frac{d v}{d t} \in L^{2}\left(0, T ; V^{\prime}\right)\right\}
$$

is a Hilbert space with respect to the inner product

$$
(u, v)_{W(0, T)}:=\int_{0}^{T}(u(t), v(t))_{V} d t+\int_{0}^{T}\left(\frac{d u(t)}{d t}, \frac{d v(t)}{d t}\right)_{V^{\prime}} d t .
$$

Moreover, if $C^{0}([0, T] ; H)$ denotes the set of continuous functions from $[0, T]$ into $H$ equipped with the norm of uniform convergence, $W(0, T) \hookrightarrow$ $C^{0}([0, T] ; H)$. Therefore any element in $W(0, T)$ has a trace $u(0) \in H$.

We now return to the initial boundary value problem (24)-(26). The idea is to apply both sides of (24) to a test function $v \in V$ and consider the resulting variational problem in $H$ for every $0<t<T$. To this end suppose $f \in L^{2}\left(0, T ; V^{\prime}\right), u_{0} \in H$, and a solution $u \in W(0, T)$ are given, then

$$
\left(\frac{d u}{d t}(\cdot), v\right)_{H}-\left(\nabla_{x} \cdot\left[F \diamond \nabla_{x} u(\cdot)\right], v\right)_{H}=(f(\cdot), v)_{H} \text { in } \mathscr{D}^{\prime}(0, T) \text { for all } v \in V .
$$

Since

$$
\left(\frac{d u}{d t}(\cdot), v\right)_{H}=\frac{d}{d t}(u(\cdot), v)_{H} \text { in } \mathscr{D}^{\prime}(0, T)
$$

for $u \in W(0, T)$ and $v \in V$, integration by parts yields

$$
\frac{d}{d t}(u(\cdot), v)_{H}+b_{\rho, k}(u(\cdot), v)=(f(\cdot), v)_{H} \text { in } \mathscr{D}^{\prime}(0, T) \text { for all } v \in V,
$$


where

$$
b_{\rho, k}(v, w):=\left(F \diamond \nabla_{x} v, \nabla_{x} w\right)_{\rho, k} \text { for } v, w \in V .
$$

Recall from Proposition 5 that there exists a constant $K(F) \leq 2 \ell$ such that if $k<K(F)$, then $b_{-1, k}(\cdot, \cdot)$, which currently is independent of $t$, satisfies Condition 1.

Condition 1. $b(t ; \cdot, \cdot): V \times V \rightarrow \mathrm{R}$ is a bilinear form for each $0 \leq t \leq T$ which satisfies

(i) for every $u, v \in V, t \mapsto b(t ; u, v)$ is measurable on $[0, T]$,

(ii) there exists an $M=M(T)>0$, independent of $t$, such that

$$
|b(t ; u, v)| \leq M\|u\|_{V}\|v\|_{V} \text { for all } t \in(0, T), u, v \in V \text {, and }
$$

(iii) there are constants $C>0$ and $\lambda$, independent of $t$ and $u$, with

$$
b(t ; u, u) \geq C\|u\|_{V}^{2}-\lambda\|u\|_{H}^{2} \text { for all } t \in[0, T], u \in V .
$$

Suppose $f \in L^{2}\left(0, T ; V^{\prime}\right)$ and $u_{0} \in H$ are given. If a function $u \in W(0, T)$ satisfies (28) with respect to the initial condition

$$
u(0)=u_{0},
$$

we call $u$ a variational solution of (24)-(26). To see why, assume $u \in W(0, T)$ is a variational solution of (24)-(26). Then (28) clearly is a weak formulation of (24). Since $u \in L^{2}(0, T ; V)$ and $V=(\mathscr{S})_{0}^{-1, k, 1},(25)$ is satisfied in the generalized sense for almost every $t \in[0, T]$. Finally, we have seen that any $u \in W(0, T)$ has a trace $u(0) \in H$, therefore (29) holds in $H=(\mathscr{S})^{-1, k, 0}$.

The following existence and uniqueness theorem follows from [4].

Theorem 4. Let $0<T<\infty, D \subset \mathrm{R}^{d}$ be an open set of finite width, and $F \in \mathscr{P}_{\ell}$ be given. Then there exists a constant $K(F) \leq 2 \ell$ such that if $\rho=-1$ and $k<K(F)$, (24)-(26) has a unique variational solution $u \in W(0, T)$ for any $f \in L^{2}\left(0, T ;\left((\mathscr{S})_{0}^{-1, k, 1}\right)^{\prime}\right)$ and $u_{0} \in(\mathscr{S})^{-1, k, 0}$. Moreover, the expectation of the solution, $E[u(\cdot)] \in L^{2}\left(0, T ; H_{0}^{1}(D)\right)$, is a variational solution of the deterministic equation that results upon replacing $F, f$, and $u_{0}$ in (24)-(26) with their respective averages.

Proof. By Proposition 5 there exists a constant $K(F) \leq 2 \ell$ such that if $k<K(F)$, the bilinear form, $b_{-1, k}(\cdot, \cdot)$, satisfies Condition 1 . The existence of a unique variational solution $u \in W(0, T)$ then follows from [4].

To prove the last statement, suppose $u \in W(0, T)$ is a variational solution of (24)-(26). Observe that

$$
\left((\mathscr{S})_{0}^{-1, k, 1}\right)^{\prime} \cong\left(H_{0}^{1}(D) \otimes(\mathscr{S})^{-1, k}\right)^{\prime} \cong H^{-1}(D) \otimes(\mathscr{S})^{1,-k} .
$$


Hence $\quad E[u(\cdot)] \in L^{2}\left(0, T ; H_{0}^{1}(D)\right), \quad E[F] \in L^{\infty}(D), \quad E[f] \in H^{-1}(D), \quad$ and $E\left[u_{0}\right] \in L^{2}(D)$. The result now follows from substituting test functions of the form $v=w H_{(0, \ldots)}$, where $w \in H_{0}^{1}(D)$, into the variational formulation (28)(29).

Attempts to obtain an explicit solution for (24)-(26) when $F=\exp ^{\diamond} W_{\phi_{x}}$ by extending the techniques used in [6], have so far been unsuccessful.

Results similar to Theorem 4 are readily obtained for other SPDEs of first order in $t$ as, for example,

$$
\frac{d u}{d t}-\Delta_{x} u+F \diamond u=f \text { and } \frac{d u}{d t}+\Delta_{x}\left(F \diamond \Delta_{x} u\right)=f \text { in } D \times(0, T) \times \mathscr{S}^{\prime},
$$

with suitable initial and boundary conditions. Note that in view of (iii) in Condition 1 the conditions on $F$ in Theorem 4 can be relaxed to $F \in \mathscr{F}_{k / 2}(D)$ for the former example.

In all of the above problems $F$ may be replaced by a measurable mapping $t \mapsto F(t)$ defined for $t \in[0, T]$, such that the associated bilinear form satisfies Condition 1. Our approach does, however, not apply to all interesting SPDEs of first order in $t$. If the problem contains a time dependent noise, there does not seem to be any simple way to obtain abstract existence and uniqueness results. The stochastic heat equation solved explicitly in [9] is an example of such a problem, another is

$$
\frac{d u}{d t}-\nabla_{x} \cdot\left(\exp ^{\diamond} W_{x, t} \diamond \nabla_{x} u\right)=0 \text { in } D \times(0, T) \times \mathscr{S}^{\prime},
$$

where $D \subset \mathrm{R}^{d}$. The problem is that $\exp ^{\diamond} W_{x, t} \in \mathscr{P}_{\ell}(D \times[0, T])$ is a formal expansion over the probability space $\left(\mathscr{S}^{\prime}\left(\mathrm{R}^{d+1}\right), \mu_{d+1}\right)$, whereas $u$ which belongs to $V=(\mathscr{S})_{0}^{-1, k, 1}(D)$ is an expansion over $\left(\mathscr{S}^{\prime}\left(\mathbf{R}^{d}\right), \mu_{d}\right)$. This means the associated bilinear form $(\omega, t) \mapsto b(t ; u, v)$ depends on $\omega \in \mathscr{S}^{\prime}\left(\mathrm{R}^{d+1}\right)$, and the approach presented above does not apply.

\section{Hyperbolic SPDEs.}

We conclude our discussion on time dependent SPDEs by indicating how one can obtain existence and uniqueness theorems for problems of second order in $t$. For simplicity we restrict our attention to a specific example that illustrates the main difference between this case and the one considered in the previous section. Suppose $0<T<\infty, D \subset \mathrm{R}^{d}$ is an open set of finite width, and $F \in \mathscr{F}_{\ell}(D)$ for some real $\ell$. Let $V=(\mathscr{S})_{0}^{\rho, k, 1}$ and $H=(\mathscr{S})^{\rho, k, 0}$ such that

$$
V \hookrightarrow H \hookrightarrow V^{\prime}
$$

is a Gel'fand triple. We intend to show 


$$
\frac{d^{2} u}{d t^{2}}-\Delta_{x} u+F \diamond u=f \text { in } D \times(0, T) \times \mathscr{S}^{\prime}
$$

has a unique variational solution with respect to the initial and boundary conditions

$$
\begin{gathered}
\left.u(t)\right|_{\partial D}=0 \text { for } 0<t<T \text { and } \\
u(0)=u_{0} ; \frac{d u}{d t}(0)=u_{1},
\end{gathered}
$$

where $f \in L^{2}(0, T ; H), u_{0} \in V$, and $u_{1} \in H$ are given.

We know from Section 4 that $L u=-\Delta_{x} u+F \diamond u$ gives rise to the bilinear form

$$
b(u, v)=b_{0}(u, v)+b_{1}(u, v),
$$

where $b_{0}(u, v):=\left(\nabla_{x} u, \nabla_{x} v\right)_{\rho, k, 0}$ contains the 'principal part' of the differential operator. The variational formulation amounts to finding a $u \in C^{0}([0, T] ; V)$ with $u^{\prime}:=d u / d t \in C^{0}([0, T] ; H)$ which satisfies

$$
\frac{d}{d t}\left(u^{\prime}(\cdot), v\right)_{H}+b(u(\cdot), v)=(f(\cdot), v)_{H} \text { in } \mathscr{D}^{\prime}(0, T) \text { for all } v \in V,
$$

and the initial conditions

$$
u(0)=u_{0} ; u^{\prime}(0)=u_{1} .
$$

To see the relation between (30)-(32) and the variational formulation, suppose $u$ is a variational solution. Then (33) is a weak formulation of (30) and (31) is satisfied in the generalized sense for almost every $t \in[0, T]$. The initial conditions $u(0)=u_{0}$ and $u^{\prime}(0)=u_{1}$ are satisfied in $V$ and $H$, respectively.

TheOREM 5. Let $0<T<\infty, D \subset \mathrm{R}^{d}$ be an open set of finite width, and $F \in \mathscr{F}_{\ell}(D)$ for some real $\ell$. If $k \leq 2 \ell$, then (30)-(32) has a unique variational solution

$$
u \in C^{0}\left([0, T] ;(\mathscr{S})_{0}^{-1, k, 1}\right) \text { with } u^{\prime} \in C^{0}\left([0, T] ;(\mathscr{S})^{-1, k, 0}\right)
$$

for any $f \in L^{2}\left(0, T ;(\mathscr{S})^{-1, k, 0}\right), u_{0} \in(\mathscr{S})_{0}^{-1, k, 1}$, and $u_{1} \in(\mathscr{S})^{-1, k, 0}$. Moreover, the expectation of the solution, $E[u(\cdot)]$, is a variational solution of the deterministic equation that results upon replacing $F, f, u_{0}$, and $u_{1}$ in (30)-(32) with their respective averages.

Proof. Let $\rho=-1$ and $k \leq 2 \ell$. Since $V \hookrightarrow H \hookrightarrow V^{\prime}$ is a Gel'fand triple, $b(\cdot, \cdot)$ is independent of $t \in[0, T], b(\cdot, \cdot)$ satisfies Condition 1 , and $b_{0}(\cdot, \cdot)$ is symmetric, the existence of a unique variational solution to (30)-(32) follows from [4]. 
The last statement follows by substituting test functions of the form $v=w H_{(0, \ldots)}$, where $w \in H_{0}^{1}(D)$, into the variational formulation.

The main difference between the assumptions here and in the previous section is that $b_{0}(\cdot, \cdot)$ has to be symmetric, that is,

$$
b_{0}(u, v)=b_{0}(v, u) \text { for all } u, v \in V .
$$

Since $(F \diamond u, v)_{-1, k, 0}$ is not symmetric when $F$ is stochastic, the equation

$$
\frac{d^{2} u}{d t^{2}}-\nabla_{x} \cdot\left(F \diamond \nabla_{x} u\right)=f \text { in } D \times(0, T) \times \mathscr{S}^{\prime},
$$

with the initial boundary conditions (31)-(32) does not satisfy (34). To see why the Wick bilinear form fails to be symmetric. Suppose that $F=\sum_{\alpha} F_{\alpha} H_{\alpha} \in(\mathscr{S})^{\rho, k}$ is stochastic, i.e., assume $F_{\gamma} \neq 0$ for some $|\gamma| \neq 0$. Let $u=u_{(0, \ldots)} H_{(0, \ldots)}$ and $v=v_{\gamma} H_{\gamma}$ where $u_{(0, \ldots)} v_{\gamma} \neq 0$, then

$$
(F \diamond u, v)_{\rho, k}=F_{\gamma} u_{(0, \ldots)} v_{\gamma}(\gamma !)^{1+\rho}(2 \mathrm{~N})^{k \gamma} \neq 0=(F \diamond v, u)_{\rho, k}
$$

for any $\rho$ and $k$.

Various generalizations are possible. But as in the previous section there seems to be no simple approach if the differential equation contains a time dependent noise. A complete treatment of the variational method for problems of second order in $t$, can be found in [4].

\section{Concluding Remarks.}

It is well known that variational solutions are stable under small perturbation of the boundary values, coefficients, or right hand side. This can, for instance, be used to prove that if $u_{\phi^{(n)}}$ is the variational solution of (14)-(15) for given $f \in(\mathscr{S})^{-1, k, 0}$ and $g \in(\mathscr{S})^{-1, k, 1}$ with respect to the noise $F_{\phi^{(n)}}=\exp ^{\diamond} W_{\phi^{(n)}}$, where $\phi^{(n)}(x):=n^{d} \phi(n x)$ for $\phi \in \mathscr{S}\left(\mathrm{R}^{d}\right)$ with $\int \phi(x) d x$ $=1$. Then $\left\|u_{\phi^{(n)}}-u\right\|_{-1,-k, 1} \rightarrow 0$ as $n \rightarrow \infty$, where $u$ is the solution of (14)(15) with respect to the singular exponential white noise $F=\exp ^{\diamond} W_{x}$.

For many of the problems we have studied it is straightforward to extend regularity results for the corresponding deterministic problems. Consider (14)-(15) on a bounded open set $D \subset \mathrm{R}^{d}$ of class $C^{2+m}$ (see [2]). From Theorem 1 there exists a unique solution $u$, with respect to $F=\exp ^{\diamond} W_{x}$ or $F_{\phi}=\exp ^{\diamond} W_{\phi_{x}}$, for any $f \in(\mathscr{S})^{-1, k, 0}$ and $g \in(\mathscr{S})^{-1, k, 1}$. Assume that $f \in(\mathscr{S})^{-1, k, m}$ and $g \in(\mathscr{S})^{-1, k, m+2}$ for some $m \in \mathrm{N}$, then $u=h+g$ where the first term of $h$ satisfies

$$
\left(\nabla h_{(0, \ldots)}, \nabla v\right)_{0, D}=\left(f_{(0, \ldots)}+\Delta g_{(0, \ldots)}, v\right)_{0, D} \text { for every } v \in H_{0}^{1}(D) .
$$

Since $f_{(0, \ldots)}+\Delta g_{(0, \ldots)} \in H^{m}(D)$, Weyl's lemma implies $h_{(0, \ldots)} \in H_{0}^{m+2}(D)$ and 
therefore $u_{(0, \ldots)} \in H^{m+2}(D)$. Assume inductively, $h_{\gamma} \in H_{0}^{m+2}(D)$ and that $h_{\beta} \in H_{0}^{m+2}(D)$ for all $\beta=\left(\beta_{1}, \beta_{2}, \ldots\right)$ such that $0 \leq \beta_{j} \leq \gamma_{j}$ for $j=1,2, \ldots$, then $h_{\gamma+\varepsilon_{i}} \in H_{0}^{m+2}(D)$. To see this note that

$$
\left(\nabla h_{\gamma+\varepsilon_{i}}, \nabla v\right)_{0, D}=\left(f_{\gamma+\varepsilon_{i}}+\Delta g_{\gamma+\varepsilon_{i}}+\sum_{\alpha+\beta=\gamma+\varepsilon_{i},|\alpha| \neq 0} \nabla \cdot\left[k_{\alpha} \nabla\left(h_{\beta}+g_{\beta}\right)\right], v\right)_{0, D},
$$

for every $v \in H_{0}^{1}(D)$. Since $k_{\alpha} \in C^{\infty}\left(\mathrm{R}^{d}\right)$ for all $\alpha$ the term on the right hand side belongs to $H^{m}(D)$ and therefore by Weyl's lemma $h_{\gamma+\varepsilon_{i}} \in H_{0}^{m+2}(D)$. If $m \geq 1+d / 2$, Sobolev's lemma implies $u$ actually is a classical solution along each fiber $H_{\gamma}$.

In all our examples we have interpreted the boundary conditions in the generalized sense, by requiring that $u-g \in(\mathscr{S})_{0}^{\rho, k, m}(D)$ for a given $g \in(\mathscr{S})^{\rho, k, m}(D)$. This approach has the advantage that we do not have to impose any regularity conditions on the domain, $D \subset \mathrm{R}^{d}$. Alternatively, we could specify the boundary data in the stochastic trace spaces

$$
(\mathscr{S})^{\rho, k, m-1 / 2}(\partial D) \cong H^{m-1 / 2}(\partial D) \otimes(\mathscr{S})^{\rho, k},
$$

for $m \in \mathrm{N}$ and bounded open sets $D \subset \mathrm{R}^{d}$.

Acknowledgements. Fred Espen Benth, Jon Gjerde, Helge Holden, Gunnar Taraldsen, Jan Ubøe, and Bernt Øksendal are thanked for valuable suggestions and encouragement during the work with this paper.

\section{Appendix.}

This section is devoted to the rather technical proof of Lemma 1. If $d=1$, the result is well known and a proof can be found in [15]. As far as we know the $d$-dimensional result has not been published although it has been applied on several occasions.

Lemma 1. If $d \geq 1$ and $k>1+\log _{2} d$, then

$$
\sum_{\alpha}(2 \mathrm{~N})^{-k \alpha}<\infty \text {. }
$$

Proof. We may write

$$
\sum_{\alpha}(2 \mathrm{~N})^{-k \alpha}=\sum_{N=0}^{\infty}\left(\sum_{\operatorname{Index~} \alpha=N}(2 \mathrm{~N})^{-k \alpha}\right),
$$

where Index $\alpha:=\max \left\{m: \alpha_{m} \neq 0\right\}$. The $N$ th term in the series on the right hand side of (35) may be expressed as 


$$
\begin{aligned}
a_{N} & :=\sum_{\operatorname{Index} \alpha=N}(2 \mathrm{~N})^{-k \alpha} \\
& =\sum_{\alpha_{1}=0}^{\infty} \cdots \sum_{\alpha_{N-1}=0}^{\infty} \sum_{\alpha_{N}=1}^{\infty} \prod_{j=1}^{N}\left(2^{d} \beta_{1}^{(j)} \beta_{2}^{(j)} \cdots \beta_{d}^{(j)}\right)^{-k \alpha_{j}} \\
& =\prod_{j=1}^{N-1} \sum_{\alpha_{j}=0}^{\infty}\left(2^{d} \beta_{1}^{(j)} \cdots \beta_{d}^{(j)}\right)^{-k \alpha_{j}} \sum_{\alpha_{N}=1}^{\infty}\left(2^{d} \beta_{1}^{(N)} \cdots \beta_{d}^{(N)}\right)^{-k \alpha_{N}} \\
& =\prod_{j=1}^{N-1}\left(\frac{\left(2^{d} \beta_{1}^{(j)} \cdots \beta_{d}^{(j)}\right)^{k}}{\left(2^{d} \beta_{1}^{(j)} \cdots \beta_{d}^{(j)}\right)^{k}-1}\right) \cdot \frac{1}{\left(2^{d} \beta_{1}^{(N)} \cdots \beta_{d}^{(N)}\right)^{k}-1}
\end{aligned}
$$

where we have used the conventions $\sum_{\operatorname{Index} \alpha=0}(2 \mathrm{~N})^{-k \alpha}=1$ and $\prod_{j=1}^{0} c_{j}=1$ for any $c_{j}$.

Since every term, $a_{N}$, in the series (35) is positive, the lemma follows if we can prove boundedness of (35). We prove boundedness by determining upper bounds on the coefficients, $a_{N}$, or equivalently lower bounds on $B_{i}:=\beta_{1}^{(i)} \cdots \beta_{d}^{(i)}$ for every $i$.

By (if necessary) reordering the basis $\left\{e_{j}\right\}_{j=1}^{\infty}$ for $L^{2}\left(\mathrm{R}^{d}\right)$ we may assume $B_{i} \leq B_{j}$ for all integers $1 \leq i \leq j$. Thus the $B_{i}$ are ordered according to size. To obtain a lower bound on $B_{i}$ we assume that $\beta_{1}^{(i)}, \ldots, \beta_{d}^{(i)}$ runs through all possible combinations of integers. Suppose $B_{i}=n=p_{1}^{\ell_{1}} p_{2}^{\ell_{2}} \cdots p_{m}^{\ell_{m}}$, where $2 \leq p_{1}<\cdots<p_{m}$ are the prime factors of $n$ and $\ell_{1}, \ldots, \ell_{m} \in \mathrm{N}$. Then $B_{i}=\beta_{1}^{(i)} \cdots \beta_{d}^{(i)}$ can be obtained in at most $d^{\ell_{1}} \cdots d^{\ell_{m}}$ distinct ways, i.e., with different $\beta_{j}^{(i)}$,s. Since $\left[\log _{2} n\right] \geq \ell_{1}+\cdots+\ell_{m}$, where [.] denotes the Gauss symbol, we find that $B_{1} \geq 1, \quad B_{i} \geq 2$ if $i>d^{\left[\log _{2} 1\right]}=1, \quad B_{i} \geq 3$ if $i>d^{\left[\log _{2} 1\right]}+d^{\left[\log _{2} 2\right]}=1+d$, etc. In general, if $n=2^{\ell}+r$ where $0 \leq r<2^{\ell}$ then $B_{i} \geq n$ whenever

$$
i>\sum_{j=1}^{n} d^{\left[\log _{2} j\right]}=1+2 d+(2 d)^{2}+\cdots+(2 d)^{\ell-1}+r d^{\ell}=\frac{(2 d)^{\ell}-1}{2 d-1}+r d^{\ell} .
$$

By defining

$$
i(n):=\frac{(2 d)^{1+\log _{2} n}-1}{2 d-1}>\frac{(2 d)^{\ell}-1}{2 d-1}+r d^{\ell},
$$

we find, by calculating the inverse of $i(n)$, that

$$
B_{i}=\beta_{1}^{(i)} \cdots \beta_{d}^{(i)} \geq n(i)=\left(\frac{1+(2 d-1) i}{2 d}\right)^{c}
$$

where $c=c(d)=\left(1+\log _{2} d\right)^{-1}$.

It follows that 


$$
a_{N} \leq b_{N}:=\prod_{j=1}^{N-1} \frac{\left(2^{d} n(j)\right)^{k}}{\left(2^{d} n(j)\right)^{k}-1} \cdot \frac{1}{\left(2^{d} n(N)\right)^{k}-1}
$$

for every positive integer $N$. Therefore the lemma follows if $\sum_{N=1}^{\infty} b_{N}<\infty$. To show convergence we apply Abel's criterion. First we compute

$$
\frac{b_{N}}{b_{N+1}}-1=\frac{(1+(2 d-1)(N+1))^{c k}-2^{-d k}(2 d)^{c k}-(1+(2 d-1) N)^{c k}}{(1+(2 d-1) N)^{c k}} .
$$

Then the identity

$$
\lim _{N \rightarrow \infty} N \frac{(1+a+N)^{t}-(a+N)^{t}}{(a+N)^{t}}=\lim _{N \rightarrow \infty} N \frac{(1+N)^{t}-N^{t}}{N^{t}}=t \text { when } t \geq 1
$$

implies

$$
\lim _{N \rightarrow \infty} N\left(\frac{b_{N}}{b_{N+1}}-1\right)=c k>1
$$

when $k \cdot c(d)>1$. Hence Abel's criterion shows

$$
\sum_{N=0}^{\infty} a_{N} \leq 1+\sum_{N=1}^{\infty} b_{N}<\infty
$$

if $k>1+\log _{2} d$, which completes proof.

\section{REFERENCES}

1. Milton Abramowitz and Irene A. Stegun, Handbook of Mathematical Functions, Dover Publications, New York, 1972.

2. Shmuel Agmon, Lectures on Elliptic Boundary Value Problems, van Norstrand mathematical studies, Princeton, New Jersey, 1965.

3, A. T. Bharucha-Reid, Random Integral Equations, Academic Press, New York, 1972.

4. Robert Dautray and Jacques-Louis Lions, Mathematical Analysis and Numerical Methods for Science and Technology, Springer-Verlag, 1985.

5. Takeyuki Hida, Hui-Hsiung Kuo, Jürgen Potthoff and Ludwig Streit, White Noise, Kluwer Academic Publishers, Dordrecht, 1993.

6. Helge Holden, Tom Lindstrøm, Bernt Øksendal, Jan Ubøe and Tu-Sheng Zhang, The Pressure Equation for Fluid Flow in a Stochastic Medium, Potential Analysis 4 (1995), 655674.

7. Helge Holden, Tom Lindstrøm, Bernt $\varnothing \mathrm{ksendal,} \mathrm{Jan} \mathrm{Ubøe} \mathrm{and} \mathrm{Tu-Sheng} \mathrm{Zhang,} \mathrm{The} \mathrm{Sto-}$ chastic Wick-Type Burgers Equation, In A. Etheridge (ed): Stochastic Partial Differential Equations, Cambridge University Press (1995), 141-161.

8. Helge Holden, Tom Lindstrøm, Bernt Øksendal, Jan Ubøe and Tu-Sheng Zhang, Stochastic Boundary Value Problems. A White Noise Functional Approach, Probab. Theory Related Fields 95 (1993), 391-419. 
9. Helge Holden, Tom Lindstrøm, Bernt Øksendal, Jan Ubøe and Tu-Sheng Zhang, The Burgers Equation with a Noisy Force and the Stochastic Heat Equation, Comm. Partial Differential Equations 19 (1 \& 2) (1994), 119-141.

10. Helge Holden, Bernt Øksendal, Jan Ubøe and Tu-Sheng Zhang, Stochastic Partial Differential Equations, Birkhäuser, 1996.

11. Yuri G. Kondratiev, Peter Leukert and Ludwig Streit, Wick Calculus in Gaussian Analysis, Acta Applicandae Mathematicae 44 (1996), 269-294.

12. Tom Lindstrøm, Bernt $\varnothing \mathrm{ksendal}$ and Jan Ubøe, Stochastic Differential Equations Involving Positive Noise, in M. Barlow and N. Bingham (eds): Stochastic Analysis, Cambridge University Press (1991), 261-303.

13. Gjermund VaŸge, Stochastic Differential Equations and Kondratiev Spaces, Ph.D. thesis, 1995.

14. Joseph Wloka, Partial Differential Equations, Cambridge University Press, Cambridge, 1987.

15. Tu-Sheng Zhang, Characterizations of White Noise Test Functions and Hida Distributions, Stochastics 41 (1992), 71-87.

DEPARTMENT OF MATHEMATICS

UNIVERSITY OF OSLO

BOX 1053 BLINDERN

$\mathrm{N}-0316$ OSLO

NORWAY 